\title{
ESTRATEGIAS DE PRODUCCIÓN MÁS LIMPIA EN EL SECTOR DE COMIDAS RÁPIDAS
}

\author{
Clean production STRATEgy \\ FOR A FAST FOOD INDUSTRY
}

\section{Laura P. Ocampo Rojas ${ }^{1}$ Lizeth del Carmen Molina Acosta ${ }^{2}$}

Universidad de La Salle, Facultad de Ingenieria, Bogotá, Colombia.

\section{RESUMEN}

En este artículo se presenta el programa de producción más limpia (PML), propuesto para una de las cadenas de comidas rápidas más grandes de Colombia, con el fin de orientarlos en la mejora de sus procesos productivos a partir del uso eficiente de sus insumos, tomando como caso de estudio una de las sucursales ubicada en la ciudad de Bogotá, por ser representativa de los procesos llevados a cabo en este sector. La metodología aplicada para el desarrollo del programa es de tipo experimental y se dividió en 2 fases: Fase 1. Diagnóstico ambiental y Fase 2. Diseño de programa de Producción más Limpia, las cuales determinarán el avance y el proceso de la formulación del PML. Se identificaron las áreas y procedimientos en los cuales se pueden realizar mejoras en el consumo de agua, energía, generación de residuos sólidos, vertimientos e intensidad sonora. Se obtuvo que, en cuanto a consumo, el de energía es el más significativo en contraste con el de agua, siendo el área más crítica la de la cocina. Se generaron 17 estrategias de PML las cuales se priorizaron teniendo como criterios el costo y tiempo de implementación, considerando además su implicación técnica, económica y ambiental. Este programa permitirá su implementación en los actuales y futuros centros de comidas rápidas similares al estudiado, logrando que adquieran responsabilidad ambiental como valor agregado a la prestación de sus servicios.

Palabras clave: comidas rápidas, impactos ambientales, producción más limpia, responsabilidad ambiental.

\section{Abstract}

We present the cleaner production program-PML proposed for one of the largest fast food chains in Colombia, in order to guide them in the improvement of their production processes from the efficient use of their inputs, taking as a case study one of the branches located in the city of Bogota for being representative of the processes carried out in this sector. The methodology applied for the development of the program is experimental and was divided into 2 phases: Phase 1: Environmental Diagnosis and Phase 2: Design of a Cleaner Production Program, which will determine the progress and the process of the formulation of the PML. The areas and procedures in which improvements in water

\footnotetext{
1 locampo02@unisalle.edu.co, https://orcid.org/0000-0002-5002-912

2 ldcmolina@unisalle.edu.co, https://orcid.org/0000-0001-8781-481
} 
consumption, energy, generation of solid waste, discharges and sound intensity can be made were identified. It was obtained that, in terms of consumption, energy is the most significant in contrast to water, being the most critical area that of the kitchen. Seventeen PML strategies were generated, which were prioritized according to the cost and time of implementation, considering also their technical, economic and environmental implications. This program will allow its implementation in current and future fast food centers similar to the one studied, making them acquire environmental responsibility as an added value to the provision of their services.

Key words: cleaner production, environmental impacts, fast food, responsibility.

\section{INTRODUCCIÓN}

El sector de las comidas rápidas experimenta un auge y una mayor competitividad empresarial, haciendo presencia en grandes mercados mediante la implementación de estrategias comerciales, mejoras en su imagen y precios, y modificación de sus sistemas operacionales y de calidad, con el interés de captar y satisfacer las necesidades de los clientes, haciendo que sus productos sean más asequibles y apetecibles; sin embargo, han descartado como estrategia de competencia su desempeño ambiental.

Gran cantidad de materia prima e insumos, son utilizados en sus procesos sin un programa de ahorro y aprovechamiento eficiente, los cuales a su vez generan subproductos que van destinados al relleno sanitario y a las redes de alcantarillado, lo que en ocasiones son motivo de problemas ambientales locales y municipales.

La PML se define como la continua aplicación de estrategias ambientalmente preventivas integradas, con un enfoque hacia la optimización de procesos, productos y servicios, para incrementar la eficiencia total e incentivar innovaciones (Solano y Vázquez, 2008) conservando materias primas, agua y energía, disminuyendo la cantidad y toxicidad de las emisiones y residuos en la fuente, minimizando el impacto ambiental y la seguridad de los productos durante todo su ciclo de vida, mejorando de esta forma la competitividad empresarial (Restrepo Gallego, 2006).
La PML, por lo tanto, debe considerarse como una estrategia empresarial de cómo los bienes y servicios deben ser producidos bajo los actuales límites tecnológicos y económicos, y no solamente como una estrategia ambiental, (Ramos-Bell \& Lorenzo-Acosta, 2017).

El siguiente artículo presenta las acciones propuestas en el sector de comidas rápidas, considerando su viabilidad técnica, ambiental y económica, a fin de prevenir y/o mitigar los impactos ocasionados, logrando un incremento eficiente en la prestación de servicios, menores costos de operación, competitividad e imagen.

\section{Metodología}

La metodología desarrollada de tipo experimental, determinó la relación causa-efecto, identificando las problemáticas del restaurante de comidas rápidas presentadas en cada estación o área de trabajo y en cada proceso, haciendo selección de instrumentos para realizar el PML y elaborar los procedimientos para obtener resultados que favorezcan a la compañía. La metodología se dividió en dos fases e involucra cada una de las actividades desarrolladas a fin de dar cumplimiento a los siguientes objetivos:

- Realizar un diagnóstico de los procesos productivos y condiciones actuales de operación. 
- Identificar los puntos y procedimientos ambientalmente críticos en la cadena productiva.

- Establecer estrategias de producción más limpia, viables técnica, económica, y ambientalmente.

Fase I. Diagnóstico ambiental: mediante visitas técnicas y a partir de listas de chequeo se identificaron las estaciones y procesos realizados.

Se realizó la caracterización de residuos sólidos por el método de cuarteo durante los días de la semana que tienen mayor demanda: martes y viernes; y días de menor demanda: lunes y domingo, generados en cada una de las estaciones del restaurante.

Para el análisis fisicoquímico de los vertimientos, se realizó un muestreo compuesto con aforo de caudal cada hora durante 16 horas de 7:00 am a 10:00 pm para el día de mayor demanda, considerando los parámetros relacionados en la Tabla 1 .

TABla 1.

Parámetros analizados a los vertimientos

\begin{tabular}{|l|l|}
\hline Parámetro & Método \\
\hline Ph & Multiparámetro \\
\hline Temperatura & Multiparámetro \\
\hline Sólidos sedimentables & Cono imhoff \\
\hline Conductividad & Multiparámetro \\
\hline Alcalinidad & Reactivo naranja de metilo- verde bromocresol \\
\hline Grasas y aceites & Gravimétrico extracción soxhle \\
\hline Color real & Espectrofométricos $(3$ longitudes de onda) \\
\hline Dbo & Incubación 5 días \\
\hline Dqo & Por reflujo abierto y volumetría \\
\hline Dureza total & Volumétrico edta \\
\hline Fosforo & Cloruro estannoso \\
\hline Sólidos suspendidos totales & Gravimétrico secado $103^{\circ} \mathrm{C}-105^{\circ} \mathrm{C}$ \\
\hline Sulfatos & Turbidímetro \\
\hline Tensoactivos & Saam (sustancias activas azul de metileno) \\
\hline Nitritos & Espectrofométricos \\
\hline Nitratos & Espectrofométricos \\
\hline Cloruros & Espectrofométricos \\
\hline & Ba \\
\hline
\end{tabular}

Fuente: Rice, Baird \& Eaton (2017)

La medición de ruido ocupacional se realizó de 7:00 am - 9:00 pm (jornada de labores) durante una hora en cada una de las estaciones del restaurante, lobby, centro de postres, servicio, parrillas, freidoras y crew room para los días de mayor y menor demanda con un sonómetro Xtech.
Se identificaron efectos e impactos ambientales por medio de las herramientas Ecobalance y Ecomapa considerando las entradas y salidas de cada proceso productivo, para las diferentes estaciones del restaurante. 
Fase 2. Implementación del PML:

Se formuló la hipótesis del PML, a partir de los resultados obtenidos en las distintas actividades de la Fase I.

El análisis de la viabilidad técnica, económica y ambiental de las estrategias de PML formuladas, se realizó con base en la guía metodológica "Oportunidades de PML en el sector hotelero y restaurantes" a partir de una serie de preguntas (Tabla 2), para cada uno de los análisis, tomando como respuesta: $\mathrm{Si}$, probablemente (P), No, No aplicable.

TABLA 2.

Preguntas para análisis de viabilidad técnica, ambiental y económica

\begin{tabular}{|c|c|c|c|}
\hline No & Técnica & Ambiental & Económica \\
\hline 1 & $\begin{array}{l}\text { ¿Puede el restaurante llevar } \\
\text { a cabo esta alternativa? }\end{array}$ & $\begin{array}{l}\text { ¿Reduce esta opción } \\
\text { el consumo de agua? }\end{array}$ & $\begin{array}{l}\text { ¿La opción está de acuerdo con la } \\
\text { política del restaurante sobre gastos } \\
\text { e inversiones? }\end{array}$ \\
\hline 2 & $\begin{array}{l}\text { ¿Puede esta opción ser llevada sin cambios } \\
\text { sustanciales en la existente infraestructura } \\
\text { del restaurante? }\end{array}$ & $\begin{array}{l}\text { ¿Reduce esta opción } \\
\text { el consumo de energía? }\end{array}$ & $\begin{array}{l}\text { ¿Tiene esta opción un periodo } \\
\text { aceptable de amortización? }\end{array}$ \\
\hline 3 & $\begin{array}{l}\text { ¿Se requiere de nuevos } \\
\text { materiales y piezas necesarias? }\end{array}$ & $\begin{array}{l}\text { ¿Reduce esta opción la cantidad } \\
\text { de materias primas utilizadas? }\end{array}$ & $\begin{array}{l}\text { ¿Reduce esta opción su gasto } \\
\text { de agua? }\end{array}$ \\
\hline 4 & $\begin{array}{l}\text { ¿Disponen los empleados de los } \\
\text { conocimientos y/o experiencia necesarios } \\
\text { para llevar a cabo esta opción? }\end{array}$ & $\begin{array}{l}\text { ¿Minimiza esta opción la cantidad } \\
\text { de aguas contaminadas? }\end{array}$ & $\begin{array}{l}\text { ¿Reduce esta opción el gasto } \\
\text { en materiales adicionales? }\end{array}$ \\
\hline 5 & $\begin{array}{l}\text { ¿Se requiere de servicios adicionales } \\
\text { a los que tiene la compañía para la } \\
\text { aplicación de esta alternativa? }\end{array}$ & $\begin{array}{l}\text { ¿Minimiza esta opción } \\
\text { la cantidad de residuos sólidos? }\end{array}$ & $\begin{array}{l}\text { ¿Reduce esta opción el gasto } \\
\text { de materias primas? }\end{array}$ \\
\hline 6 & $\begin{array}{l}\text { ¿Se conocen los responsables de la aplicación } \\
\text { y sostenimiento de esta alternativa? }\end{array}$ & $\begin{array}{l}\text { ¿Reduce esta opción la cantidad } \\
\text { de accidentes relacionados } \\
\text { con el trabajo? }\end{array}$ & $\begin{array}{l}\text { ¿Reduce esta opción el gasto } \\
\text { de disposición de residuos sólidos? }\end{array}$ \\
\hline 7 & $\begin{array}{l}\text { ¿Con esta estrategia se mejoran o mantiene } \\
\text { la calidad del componente al que pertenece? }\end{array}$ & $\begin{array}{l}\text { ¿Ayuda a mejorar la imagen } \\
\text { corporativa de la cadena } \\
\text { de comidas rápidas }\end{array}$ & $\begin{array}{l}\text { ¿Reduce esta opción el gasto } \\
\text { en control de aguas residuales? }\end{array}$ \\
\hline
\end{tabular}

Fuente: Alcaldía Mayor de Bogotá (2004).

\section{RESUlTADOS Y DISCUSIÓN}

\subsection{Estaciones y procesos}

Las siguientes son las estaciones existentes en el restaurante de comidas rápidas objeto de estudio, y son representativas de los demás restaurantes de la cadena.

- Centro de postres: área de $48 \mathrm{~m}^{2}$ donde se elaboran y venden helados; dispone de máquina de helados, avisos publicitarios, aderezos y embalaje para la preparación de postres; se generan residuos aprovechables y orgánicos, y un alto consumo de energía.
- Servicio: existen diferentes estaciones dentro de ella, como la estación de cajas, de bebidas, de papas y de domicilios. En esta área se hace la toma y entrega de pedidos a los clientes; se generan residuos sólidos y líquidos, consumo de energía y agua.

- Cocina: consta de dos espacios para la preparación de los productos ofrecidos (línea 1 y 2). En esta área de $29 \mathrm{~m}^{2}$, se encuentran diferentes estaciones: iniciador, parrilla, ensamble, y empanizado, los cuales son para la preparación de las hamburguesas y pollo apanado; la línea 2 es la estación para la 
preparación de almuerzos, y, así mismo, se encuentra la maquinaria para productos fritos y neveras auxiliares. Presenta el mayor consumo de energía, también se generan residuos sólidos orgánicos.

- Bodega: área de $8.69 \mathrm{~m}^{2}$ donde se encuentran aquellos productos que pueden permanecer a temperatura ambiente como: alimentos, embalajes y en módulos exclusivos para productos de aseo, cuenta con luces automáticas, por lo que es un área que demanda bajo consumo de energía.

- Cuartos fríos: corresponde al refrigerador y la cava que cuentan con una temperatura entre $1^{\circ} \mathrm{C}$ a $4^{\circ} \mathrm{C}$ y $-18^{\circ} \mathrm{C}$ a $-23^{\circ} \mathrm{C}$ respectivamente, donde en un área de $15.40 \mathrm{~m}^{2}$, se almacenan alimentos perecederos como lácteos, verduras, carnes, pollo, y salsas, por lo que demanda un alto consumo de energía.

- Back room: área de $5.17 \mathrm{~m}^{2}$ donde se encuentran un lavamanos para los empleados, las piletas de lavado de utensilios, trampa de grasas, lavadora, y lava traperos. Consume agua, genera vertimientos, residuos aprovechables, ordinarios y peligrosos (remanente de la trampa de grasas).

- Crew-room: área de $22.5 \mathrm{~m}^{2}$, donde los empleados toman su tiempo de descanso, cuenta, con dos baños, un vestier, casilleros, televisor y computador que consume aproximadamente de 0,437 kWh, donde se genera un alto consumo de energía y generación de residuos aprovechables y orgánicos.

- Área de comedor (lobby): área de $251.26 \mathrm{~m}^{2}$ donde los clientes hacen el consumo de los productos ofrecidos por el restaurante. Cuenta con tres canecas para la disposición de residuos, mesa de aderezos y baños al servicio de los clientes. Es el área con mayor generación de residuos sólidos y gran porcentaje de iluminación LED por lo que demanda un consumo considerable de energía.

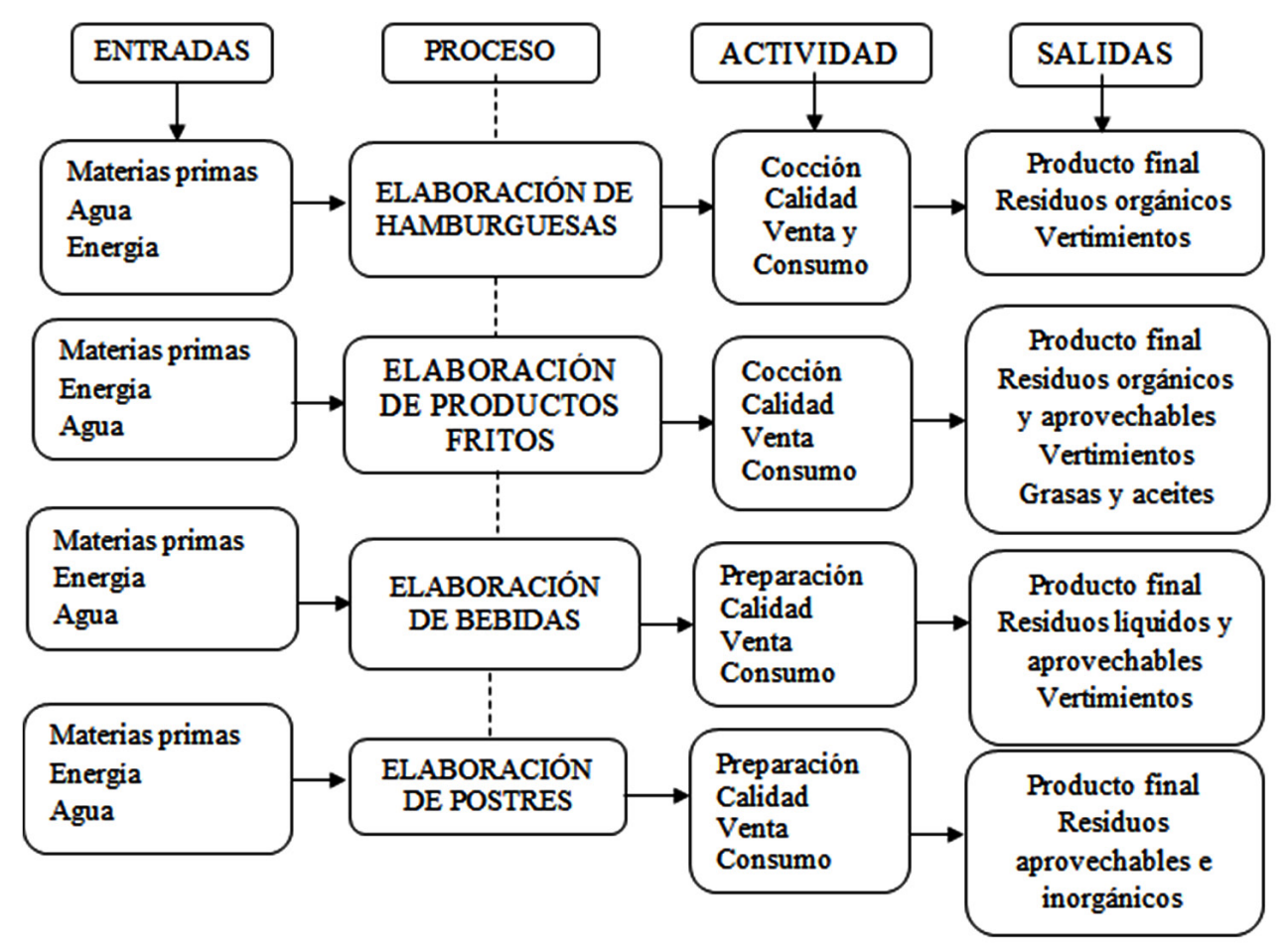

Fig. 1. Proceso productivo para la preparación de alimentos en el restaurante de comidas rápidas. Fuente: autoras 


\subsection{Caracterización de residuos sólidos}

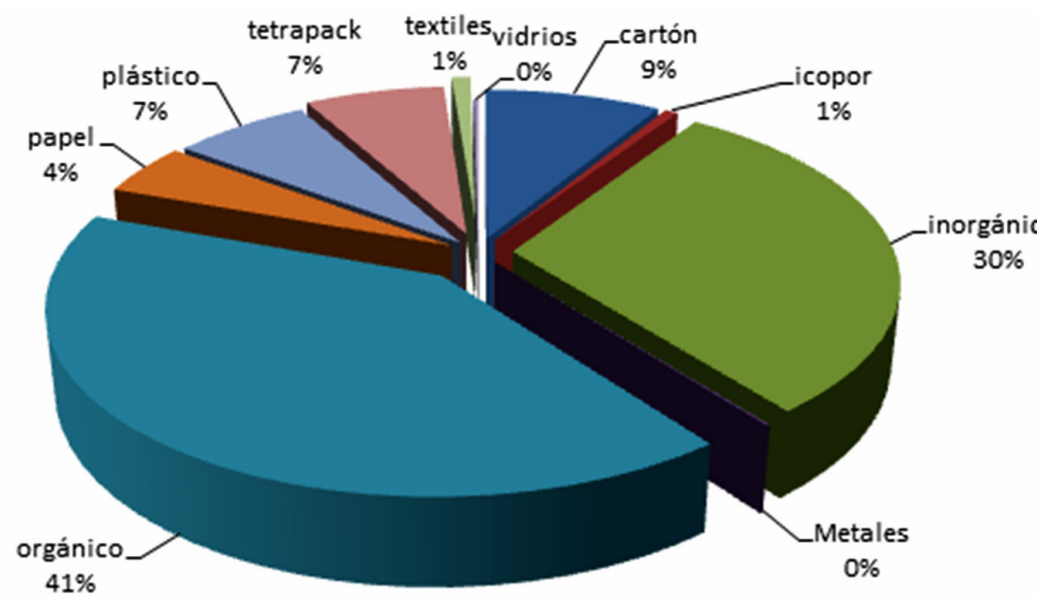

Fig. 2. Caracterización de residuos. Fuente: autoras

El residuo de mayor generación es el orgánico. Proviene de los sobrantes de alimentos vendidos y del control de calidad realizado a los ingredientes. Según
Velázquez, Cherrett \& Holdsworth (2015), este residuo presenta un alto potencial de aprovechamiento en la producción de biocombustibles y abono orgánico.

\subsection{Análisis fisicoquímico de vertimientos}

TABLA 3.

Análisis fisicoquímico de vertimientos

\begin{tabular}{|c|c|}
\hline Parámetro & Valor \\
\hline Aceites y grasas & $113 \mathrm{Mg} / \mathrm{l}$ \\
\hline Color & $77 \mathrm{Upc}$ \\
\hline Dbo5 & $313 \mathrm{Mg} / \mathrm{l}$ \\
\hline Dqo & $741 \mathrm{Mg} / \mathrm{l}-\mathrm{o} 2$ \\
\hline Dureza total & $42 \mathrm{Mg} / \mathrm{l} \mathrm{caco} 3$ \\
\hline Fósforo & $17,3 \mathrm{Mg} / \mathrm{l} \mathrm{p}$ \\
\hline Cloruros & $20 \mathrm{Mg} / \mathrm{ll}-$ \\
\hline Nitritos & $5 \mathrm{Mg} / \mathrm{l}$ \\
\hline Nitratos & $0,9 \mathrm{Mg} / 1$ \\
\hline Tensoactivos anionicos & $1,0 \mathrm{Mg} / \mathrm{l}$ \\
\hline Tensoactivos cationicos & $0,07 \mathrm{Mg} / \mathrm{l}$ \\
\hline Sólidos suspendidos totales & $152 \mathrm{Mg} / \mathrm{l}$ \\
\hline Sulfatos & $55 \mathrm{Mg} / \mathrm{l} \mathrm{so} 4$ \\
\hline Ph & $6,51 \mathrm{~A} 8,64$ \\
\hline Sólidos sedimentables & $0,2 \mathrm{~A} 6,0 \mathrm{ml} / \mathrm{l}$ \\
\hline Alcalinidad & $25 \mathrm{~A} 130 \mathrm{mg} / \mathrm{l} \mathrm{caco3}$ \\
\hline
\end{tabular}

Fuente: autoras
TABLA 4.

Aforo de caudal en el día de mayor demanda

\begin{tabular}{|c|c|}
\hline Hora & Caudal $(\mathbf{1} / \mathbf{s})$ \\
\hline $7: 00$ & 0.06 \\
\hline $8: 00$ & 0.06 \\
\hline $9: 00$ & 0.15 \\
\hline $10: 00$ & 0.22 \\
\hline $11: 00$ & 0.22 \\
\hline $12: 00$ & 0.37 \\
\hline $13: 00$ & 0.18 \\
\hline $14: 00$ & 0.20 \\
\hline $15: 00$ & 0.40 \\
\hline $16: 00$ & 0.39 \\
\hline $17: 00$ & 0.21 \\
\hline $18: 00$ & 0.18 \\
\hline $19: 00$ & 0.05 \\
\hline $20: 00$ & 0.01 \\
\hline $21: 00$ & 0.09 \\
\hline $22: 00$ & 0.08 \\
\hline
\end{tabular}

Fuente: autoras 
El agua residual generada proviene de los baños del área del lobby y crew-room, cocina, y cuartos de aseo.

Se observan altas concentraciones de DQO y DBO, debido a la materia orgánica presente. Los cuartos de aseo disponen de sifones cuyos diseños en forma rectangular y con una mayor distancia entre las divisiones de éstos, elevan los componentes orgánicos.

La presencia de sulfatos, aunque baja, indican una alta concentración de sólidos disueltos; así mismo, se destaca la relación directa que existe entre este parámetro y la conductividad y cloruros del vertimiento; (UCPYPFE, 2010).

\subsection{Medición de ruido ocupacional}

\section{Niveles de presión sonora}

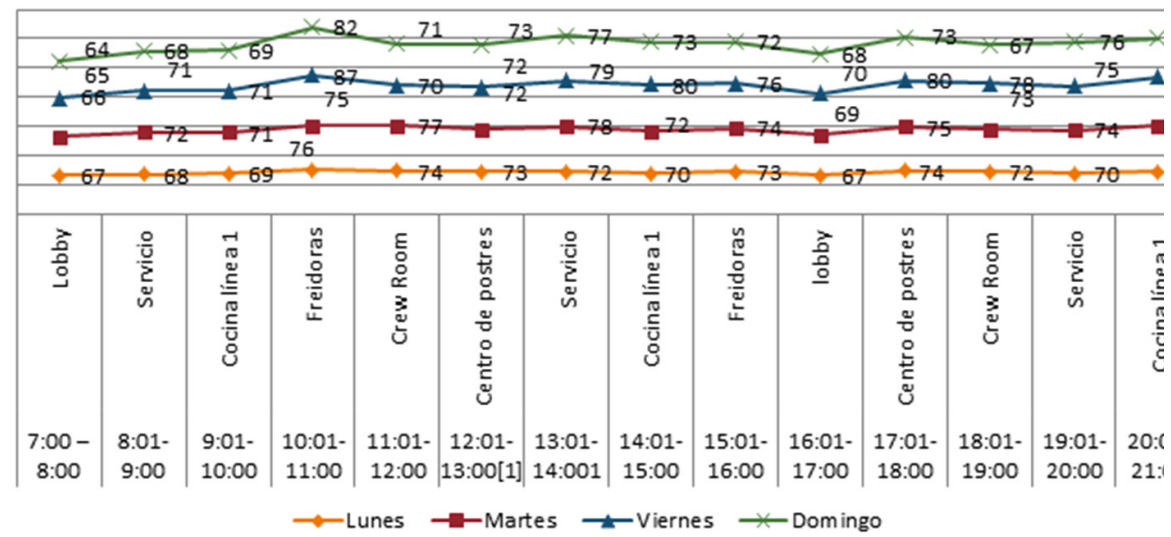

Fig. 3. Niveles de presión sonora (dBA) por área - hora

Fuente: Autoras

Los niveles de ruido por estación no superan los límites de la Resolución 1792 de 1990, sin embargo, se presentan valores que para un trabajador que labore 8 horas en la estación de freidoras, podría afectar su salud auditiva.

\subsection{Identificación de puntos criticos $y$ problemáticas ambientales}

En el Ecomapa (Fig. 4) se identifican los puntos críticos representados con los logos indicados en la Tabla 5.

TABla 5.

Convenciones empleadas en el Ecomapa

\begin{tabular}{|l|c|}
\hline \multicolumn{2}{|c|}{ Convenciones } \\
\hline Consumo de Agua & \\
\hline Generación de vertimientos & \\
\hline Consumo de Energía & \\
\hline Generación de Residuos & \\
\hline Generación de Residuos & \\
\hline Almacenamiento de residuos & \\
\hline
\end{tabular}

Fuente: autoras 


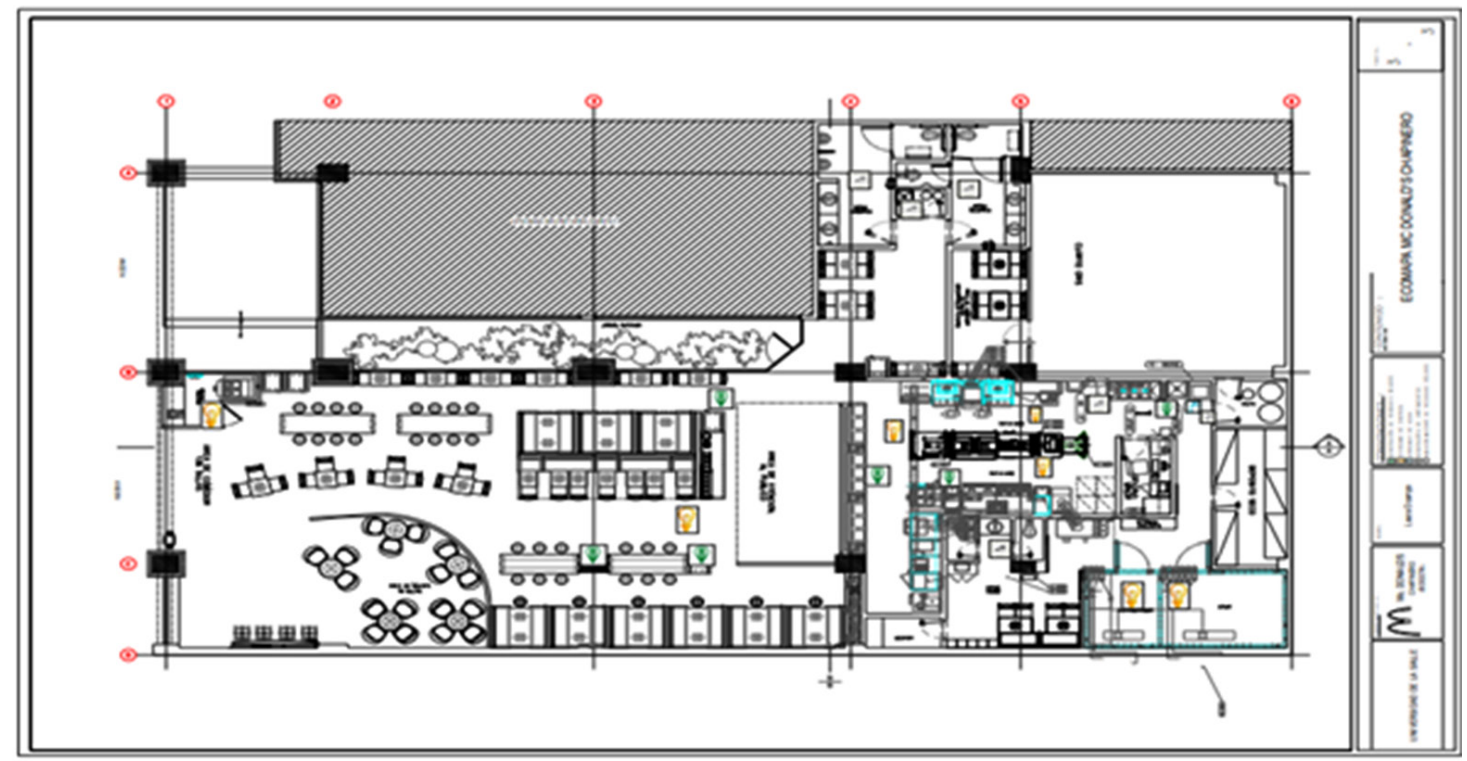

Fig. 4. Niveles de presión sonora (dBA) por área - hora. Fuente: autoras

Las áreas que presentan mayor impacto son la cocina y el centro de postres ya que representan y concentran los procesos productivos.

El Ecobalance permitió realizar un balance de entradas y salidas con enfoque en las materias primas, insumos, energía, aditivos y residuos con un alcance de los procesos genéricos de los restaurantes de comidas rápidas. Se observó que la mayoría de materias primas quedan dentro del proceso, sin embargo, los aditivos como embalajes, vasos, tapas y empaques de las materias primas, terminan como residuos sólidos, que no son separados en la fuente para una futura comercialización, y son dispuestos en el relleno sanitario a excepción del aceite vegetal para frituras, el cual, cuando ya no cumple las condiciones específicas para su uso, es almacenado en bidones con capacidad de 1 galón, y posteriormente es recolectado por una compañía autorizada que se encarga de reciclarlo y convertirlo en biodiesel o para la fabricación de jabones.
Para el componente hídrico, con base en la entrada de agua se obtiene el mismo valor de vertimientos como salida, puesto que es únicamente para el lavado de utensilios, ya que para la cocción de alimentos se hace a partir de las freidoras y parrillas con las que cuenta el restaurante, y al no hacerse uso del recurso, no existen pérdidas de este.

Respecto a la energía, se presentan altos consumos por parte de la maquinaria, por lo tanto, de forma general, se debe enfatizar en la minimización del consumo energético, generación de residuos sólidos y residuos especiales (remanente de grasa).

\subsection{Formulación del programa PML}

Para cada problemática identificada se plantearon diferentes estrategias de PML. En la Tabla 6 se especifican las acciones a desarrollar en cada una de las 17 estrategias resultantes. 
TABla 6.

Formulación de estrategias PML.

\begin{tabular}{|c|c|c|}
\hline Componente & Problemática identificada & Estrategia \\
\hline \multirow{2}{*}{ Hídrico } & $\begin{array}{l}\text { Uso aproximado de } 50 \text { litros } \\
\text { de agua en lavado de pisos }\end{array}$ & $\begin{array}{l}\text { 1. Almacenamiento de agua por condensación de aires } \\
\text { acondicionados en los tanques de agua para lavado } \\
\text { de pisos y riego de plantas }\end{array}$ \\
\hline & $\begin{array}{l}\text { Alto contenido de grasas } \\
\text { y aceites en vertimientos }\end{array}$ & $\begin{array}{l}\text { 2. Limpieza de trampa de grasas } 3 \text { veces por semana, } \\
\text { donde el remanente será almacenado en bidones } \\
\text { de } 1 \text { galón y entregado a empresas autorizadas }\end{array}$ \\
\hline \multirow{3}{*}{ Energía } & \multirow{2}{*}{$\begin{array}{l}\text { Alto consumo de energía } \\
\text { por iluminación }\end{array}$} & $\begin{array}{l}\text { 3. Instalación de sensores automáticos, en } \\
\text { áreas de crew-room, cuartos de aseo, y baños }\end{array}$ \\
\hline & & $\begin{array}{l}\text { 4. Instalación de celdas solares para la iluminación } \\
\text { de los centros de postres y automac }\end{array}$ \\
\hline & Uso de tubos T8 convencionales & $\begin{array}{l}\text { 5. Cambio de iluminación con mercurio por iluminarias } \\
\text { Led o tubos con menor contenido de mercurio } \\
\text { y entregadas directamente a la compañía autorizada } \\
\text { para su disposición final }\end{array}$ \\
\hline \multirow{10}{*}{ Residuos sólidos } & \multirow{2}{*}{$\begin{array}{l}\text { Alta generación de } \\
\text { residuos inorgánicos }\end{array}$} & $\begin{array}{l}\text { 6. Venta de icopor, y desechables (platos, cubiertos, pitillos) } \\
\text { a empresas de reciclaje }\end{array}$ \\
\hline & & $\begin{array}{l}\text { 7. Venta de plásticos con bajo nivel de contaminación } \\
\text { empresas de reciclaje }\end{array}$ \\
\hline & \multirow[t]{2}{*}{$\begin{array}{l}\text { Carencia de separación } \\
\text { en la fuente de residuos }\end{array}$} & $\begin{array}{l}\text { 8. Ubicación de puntos ecológicos internos del restaurante } \\
\text { fabricados a partir de tetrapack para los residuos: tetrapack, } \\
\text { inorgánicos, orgánicos, plásticos }\end{array}$ \\
\hline & & 9. Puntos ecológicos externos para residuos inorgánicos \\
\hline & $\begin{array}{l}\text { Desperdicio y alta generación } \\
\text { de papel publicitario }\end{array}$ & $\begin{array}{l}\text { 10. Venta de cartón y papel publicitario con bajo } \\
\text { nivel de contaminación a empresas de reciclaje }\end{array}$ \\
\hline & $\begin{array}{l}\text { Desperdicio en generación } \\
\text { de cajitas felices consumidas } \\
\text { dentro del restaurante }\end{array}$ & 11. Entrega de menú infantil sin caja de cartón \\
\hline & $\begin{array}{l}\text { Incorrecta separación } \\
\text { de residuos peligrosos }\end{array}$ & $\begin{array}{l}\text { 12. Entrega de tintas y solventes de cajas } \\
\text { registradoras a empresa autorizadas }\end{array}$ \\
\hline & $\begin{array}{l}\text { Mala gestión en disposición final } \\
\text { de residuos generados en la trampa } \\
\text { de grasas }\end{array}$ & $\begin{array}{l}\text { 13. Recolección y almacenamiento en bidones de remanente } \\
\text { de trampa de grasas y entregados empresas autorizadas }\end{array}$ \\
\hline & \multirow[b]{2}{*}{$\begin{array}{l}\text { Generación de residuos inorgánicos } \\
\text { a causa de vasos de papel }\end{array}$} & 14. Venta de vasos reutilizables a clientes \\
\hline & & $\begin{array}{l}\text { 15. Vaso - termo como obsequio a empleados } \\
\text { de los restaurantes, para su uso durante el break }\end{array}$ \\
\hline \multirow[b]{2}{*}{ Intensidad sonora } & \multirow{2}{*}{$\begin{array}{l}\text { Altos niveles de intensidad } \\
\text { sonora en horario diurno }\end{array}$} & 16. Cambio en el volumen a equipos de cocina \\
\hline & & $\begin{array}{l}\text { 17. Instalación de luces Led para aviso } \\
\text { de cocción de alimentos }\end{array}$ \\
\hline
\end{tabular}




\subsection{Análisis de la viabilidad:}

Basado en la formulación de las preguntas de la Ta- las cuales 13 de las 17 estrategias resultaron viables, bla 2, se realizó la calificación de cada estrategia, de técnica, económica y ambientalmente.

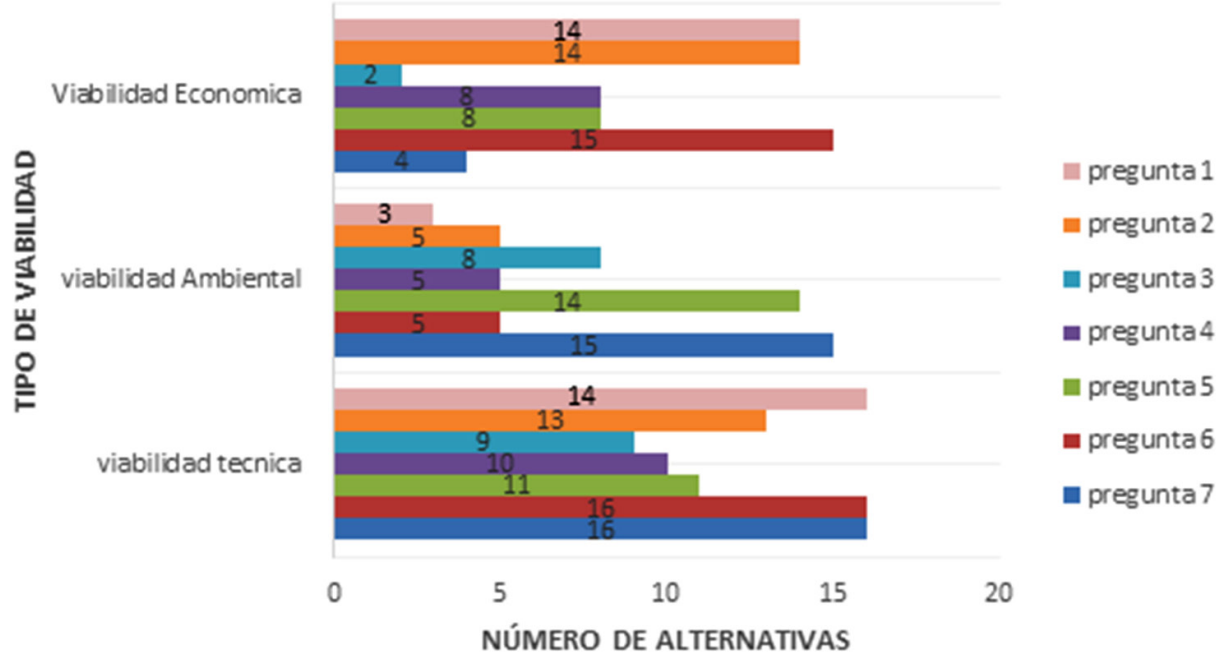

Fig. 5. Estrategias viables T.E.A

Fuente: autoras

\section{Conclusiones}

- El diagnóstico ambiental determinó que el componente energético es aquel que genera mayor impacto, teniendo en cuenta que el consumo promedio de energía es de $13.385 \mathrm{Kw} / \mathrm{h}$ a causa de los equipos de cocina, en contraste con el componente hídrico, siendo el de menor impacto, pues se hace uso de un gran número de dispositivos ahorradores.

- Los índices de generación de los residuos son proporcionales a la demanda que presenta el restaurante, agregando que la empresa durante el año presentará picos de generación, debido a las promociones realizadas y meses de menores demandas.

- A partir del Ecomapa, como herramienta de PML, se identificó el área de cocina como la más crítica del restaurante, puesto que en esta área se presenta el mayor número de equipos con gran consumo de energía y generación de residuos, seguido del lobby; adicionalmente el mayor consumo de agua se presenta en el área del backroom, ya que se realiza limpieza de utensilios, uso de la lavadora para las toallas de limpieza y lavado de manos para los empleados.

- Se observó que el nivel de presión sonora es crítico con valores superiores a $80 \mathrm{~dB}$, entre las 12 del mediodía y 6 de la tarde, en áreas como la cocina, freidoras, centro de postres, por lo que es necesario establecer medidas de mitigación con el fin de prevenir lesiones auditivas ocupacionales.

- Se generaron 17 estrategias de PML las cuales se priorizaron teniendo como criterio su costo y tiempo de implementación, así mismo se tuvo en cuenta su implicación técnica, económica y ambiental para una futura implementación, a beneficio económico y ambiental de la compañía. 


\section{REFERENCIAS}

Alcaldía Mayor de Bogotá (2004). Oportunidades de producción más limpia en el sector hotelero y servicios de restaurante. Guía para empresarios. Recuperado en: http://ambientebogota.gov.co/documents/24732/3988003/Oportunidades+de+produccion+mas +limpia+en+el+sector+hotelero+y+servicios+de+restaurante.pdf

Ministerio de Trabajo y Seguridad Social y Salud. (1990). Resolución 1792 de 1990. Por el cual se adoptan valores límites permisibles para la exposición ocupacional al ruido. Recuperado en: https://www.icbf.gov.co/cargues/avance/docs/resolucion_ minsalud_r1792_90.htm

Ramos-Bell, S. \& Lorenzo-Acosta, Y. (2017). Acciones de producción más limpia para implementar en la industria azucarera cubana. ICIDCA Sobre los Derivados de la Caña de Azúcar , 51(1), 60-66.

Restrepo Gallego, M. (2006). Producción más limpia en la industria alimentaria. Producción + Limpia, 1(1), 87-101. Rice, E.,
Baird, R. B. \& Eaton, A. D. (2017). Standard methods: for the examination of water and wastewater. $22^{\text {nd }} . e d$. Washington: American Public Health Association, American Water Works Association and Water Environmental Federation.

Solano, G. \& Vásquez, P. (2008). Manual de producción más limpia para el subsistema de salud de las Fuerzas militares. Bogotá: Dirección General de Sanidad Militar Subsistema de Salud de las Fuerzas Militares.

UCPYPFE. (2010). Informe de aavance. Proyectos sanitarios de potabilización. Recuperado en: http://www.ucpypfe.gov.ar/BirfPIHNG/IEA-PmpaAnexo3.pdf

Velazquez, A., Cherrett, T. \& Holdsworth, P. (2015). Waste-to-fuel opportunities for British quick service restaurants: A case study. Resources, Conservation and Recycling, 104(part A), 239-253. 
